Bundesgesundheitsbl 2013 - 56:1165-1165 DOI 10.1007/s00103-013-1812-4

๑) Springer-Verlag Berlin Heidelberg 2013
Empfehlung des Umweltbundesamtes

\section{Mitteilung zur Änderung der Leitlinie zur hygienischen Bewertung von Schmierstoffen im Kontakt mit Trinkwasser (Sanitärschmierstoffe) [Schmierstoffleitlinie ${ }^{1}$}

Das Umweltbundesamt plant die Überführung der Schmierstoffleitlinie in eine Bewertungsgrundlage nach dem geäntungsgrundlage wird keine Listung von Schmierstoffen mehr vorsehen.

Die Schmierstoffleitlinie und die komplette Liste der positiv geprüften Schmierstoffe sind auf der Internetseite des Umweltbundesamtes (http://www. umweltbundesamt.de/wasser/themen/ trinkwasser/schmierstoffleitlinie.htm) abrufbar.

Die folgenden Änderungen der Anlage 4 der Leitlinie entsprechen dem Stand vom 03.06.2013:

Für den Schmierstoff „Castrol Optisil Leb 2" verlängert sich die Gültigkeit des Prüfzeugnisses bis zum 01.02.2017.

Für den Schmierstoff „Geralyn 2“ verlängert sich die Gültigkeit des Prüfzeugnisses bis zum 13.02.2017. derten $₫ 17$ (3) TrinkwV 2001. Die Bewer-
Für den Schmierstoff „Silikonfett Type 2 “ verlängert sich die Gültigkeit des Prüfzeugnisses bis zum 08.12.2014.

Der Schmierstoff „Berulub SIHAF 1“ der Firma „Carl Bechem GmbH“ wird für die Anwendungsbereiche Kalt- und Warmwasser aufgenommen. Das Prüfzeugnis gilt bis zum 01.02.2018.

Der Schmierstoff „Unisilkon LCA 3801“ der Firma „Klüber Lubrication München KG“ wird für die Anwendungsbereiche Kalt- und Heißwasser aufgenommen. Das Prüfzeugnis gilt bis zum 25.06.2015.

Die Schmierstoffe „Armaturenfett Diamant Type 4“, „Chemplex 746 $(, 169)$ weiß“, „Gleitmo 815“, „Ideal Standard 12“ und „Unisilkon NCA 3001“ werden gestrichen.

\footnotetext{
${ }^{1}$ zuletzt geändert: BundesgesundheitsblGesundheitsforsch-Gesundheitsschutz 2012 55:152
} 